\title{
Document-Level Definition Detection in Scholarly Documents: Existing Models, Error Analyses, and Future Directions
}

\author{
Dongyeop Kang $^{\curvearrowright}$ Andrew Head $^{\odot}$ Risham Sidhu $^{\odot}$ \\ Kyle Lo $^{\diamond}$ Daniel S. Weld ${ }^{\diamond \diamond}$ Marti A. Hearst ${ }^{\diamond}$ \\ ${ }^{\bigcirc}$ University of California, Berkeley, ${ }^{\diamond}$ Allen Institute for AI, ${ }^{\circ}$ University of Washington \\ \{dongyeopk, andrewhead, rishamsidhu, hearst\}@berkeley.edu \\ \{kylel, danw\}@allenai.org
}

\begin{abstract}
The task of definition detection is important for scholarly papers, because papers often make use of technical terminology that may be unfamiliar to readers. Despite prior work on definition detection, current approaches are far from being accurate enough to use in realworld applications.

In this paper, we first perform in-depth error analysis of the current best performing definition detection system and discover major causes of errors. Based on this analysis, we develop a new definition detection system, HEDDEx, that utilizes syntactic features, transformer encoders, and heuristic filters, and evaluate it on a standard sentence-level benchmark. Because current benchmarks evaluate randomly sampled sentences, we propose an alternative evaluation that assesses every sentence within a document. This allows for evaluating recall in addition to precision.

HEDDEx outperforms the leading system on both the sentence-level and the document-level tasks, by $12.7 \mathrm{~F} 1$ points and $14.4 \mathrm{~F} 1$ points, respectively. We note that performance on the high-recall document-level task is much lower than in the standard evaluation approach, due to the necessity of incorporation of document structure as features. We discuss remaining challenges in document-level definition detection, ideas for improvements, and potential issues for the development of reading aid applications.
\end{abstract}

\section{Introduction}

Automatic definition detection is an important task in natural language processing (NLP). Definitions can be used for a variety of downstream tasks, such as ontology matching and construction (Bovi et al., 2015), paraphrasing (Hashimoto et al., 2011), and word sense disambiguation (Banerjee and Pedersen, 2002; Huang et al., 2019). Prior work in au-

\begin{abstract}
Example
$\underline{s}^{\text {task }}$ are softmax-normalized weights and the scalar [...]

Textual entailment is the task of determining whether a "hypothesis" is true, given a "pre-mise",

A biLM combines both a forward and backward LM

[...] a fine grained word sense disambiguation (WSD) task and a POS tagging $\overline{\text { task }}$.
\end{abstract}

Table 1: Examples of terms and definitions from Peters et al. (2018). Each row shows a term (e.g., $\left.s^{\text {task }}\right)$ along with its definition (e.g., "softmax-normalized weights").

tomated definition detection has addressed the domain of scholarly articles (Reiplinger et al., 2012; Jin et al., 2013; Espinosa-Anke and Schockaert, 2018; Vanetik et al., 2020; Veyseh et al., 2020). Definition detection is especially important for scholarly papers because they often use unfamiliar technical terms that readers must understand to properly comprehend the article.

In formal terms, definition detection is comprised of two tasks: classifying sentences as containing definitions or not, and identifying which spans within these sentences contain terms and definitions. As the performance of definition extractors continues to improve, these algorithms could pave the way for new types of intelligent assistance for readers of dense technical documents. For example, one could envision future interfaces that reveal definitions of jargon like "biLM" or the symbol "s $s$ task" when a reader hovers over the terms in a reading application (Head et al., 2020). Examples of sentences containing terms and definitions are shown in Table 1.

Despite recent advances in definition detection, 
much work remains to be done before models are capable of extracting definitions with an accuracy appropriate for real-world applications. The first challenge is one of recall: existing systems are typically not trained to identify all definitions in a document, but rather to classify individual sentences arbitrarily sampled from a large corpus. The second challenge is one of precision: the state of the art misclassifies upwards of $30 \%$ of sentences (Veyseh et al., 2020). This begs the questions of why definition extractors fall short, and how these shortcomings can be overcome.

In this paper, we contribute the following:

- An in-depth error analysis of the current bestperforming model. This analysis characterizes the state of the field and illustrates future directions for improvement;

- A new model, Heuristically-Enhanced Deep Definition Extraction (HEDDEx), that extends a state-of-the-art model with improvements designed to address the problems found in the error analysis. An evaluation shows that this improved model outperforms the state of the art by a large margin $(+12.7 \mathrm{~F} 1)$;

- An introduction of the challenging task of fulldocument definition detection. In this task, models are evaluated based on their ability to identify definitions across an entire document's sentences. We believe this framing of definition detection is critical to preparing future algorithms for real-world use;

- A preliminary analysis of previous models and our model on the document-level definition detection task using a small test set of scholarly papers where every term and definition has been labeled. This analysis shows that HEDDEx outperforms the state of the art, while revealing opportunities for future improvements.

In summary, this paper draws attention to the work yet to be done in addressing the task of document-level definition detection for scholarly documents. We draw attention to the fact that a seemingly straightforward task like definition detection still poses significant challenges to NLP, and that this is an area that needs more focus in the scholarly document processing community.

\section{Related Work}

Definition detection has been tackled in several ways in prior research. The traditional rule-based systems (Muresan and Klavans, 2002; Westerhout and Monachesi, 2008; Westerhout, 2009a) used hand-written definition patterns (e.g., "is defined as") and linguistic features (e.g., pronoun, verb, punctuation), providing high precision but low recall detection. To address the low recall problem, model-driven approaches (Fahmi and Bouma, 2006; Westerhout, 2009b; Navigli and Velardi, 2010; Reiplinger et al., 2012) were developed using statistical and syntactic features such as bagof-words, sentence position, part-of-speech (POS) tags, and their combination with hand-written rules. Notably, Jin et al. (2013) used conditional random field (CRF) (Lafferty et al., 2001) to predict tags of each token in a sentence such as TERM for term tokens, DEF for definition tokens, and 0 for neither. Recently, sophisticated neural models such as convolutional networks (Espinosa-Anke and Schockaert, 2018) and graph convolutional networks (Veyseh et al., 2020) have been applied to obtain better sentence representations in combination with syntactic features. However, our analysis found that the state-of-the-art is still far from solving the problem, achieving an F1 score of only 60 points on a standard test set.

\section{Error Analysis of the Leading System}

In order to inform our efforts to develop a more advanced system, we performed an in-depth error analysis of the results of the current leading approach to definition and term identification, the joint model by Veyseh et al. (2020). We analyzed the models' predictions on the W00 dataset (Jin et al., 2013) since it matches our target domain of scholarly papers and is the dataset that the joint model was evaluated on. Of the 224 test sentences, ${ }^{1}$ the Veyseh et al. (2020) system got 111 correct. The first author annotated the remaining 113 sentences for which the algorithm was partially or fully incorrect to ascertain the root causes of the errors.

We discovered four (for terms) and five (for definitions) major causes for the erroneous predictions, as summarized in Table 3. We illustrate three exam-

\footnotetext{
${ }^{1}$ Note that we use the test set of W00 for manual analysis, which is only $10 \%$ of the entire dataset. In our experiment in $\S 4$, we didn't use the test set used in this error analysis, but did cross-validation using the train set, following the experimental setup in Veyseh et al. (2020).
} 


\begin{tabular}{llll}
\hline Sentences & Cause & Patterns & Solutions \\
\hline [Equal] is open in something of type collection where that & $\bullet$ Overgeneralization: & $\begin{array}{l}\text { (none } \\
\text { collection is a \{partition of something\} }\end{array}$ & $\begin{array}{l}\text { technical term bias } \\
\bullet \text { description (is) }\end{array}$
\end{tabular}

A [graph - based operator] defines a transformation on a multi-document graph (MDG) $\bar{G}^{-}$which preserves lsome of its properties while reducing the number\}...

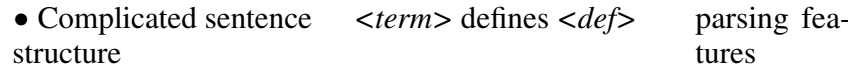

- Unfamiliar or unseen

vocabulary

- Unseen patterns
$<$ term $>$ that we have developed enables us to automatically $<$ def $>$ generalize patterns

we have developed enables us to automatically extract a corpus $\mathrm{N}-\mathrm{V}$ pairs whose elements axe linked by one of the semantic relations defined in the qualia structure ...

Table 2: Sample annotations from our analysis of errors produced by Veyseh et al.'s (2020) joint model when extracting definitions from the WQQ (Jin et al., 2013) dataset. Each row includes a sentence annotated with gold labels for terms and definitions, and the system's predictions for [terms] and \{definitions $\}$ ("Sentences"). Also shown is a class of error ("Cause"), surface patterns that we anticipate could be used to correct the detection of the definition ("Patterns"), and classes of improvements to make to the model ("Solutions"). The first row is an example of a false positive; the second row is a partially-correct prediction; and the third row is a false negative. A transcription error ('axe' instead of 'are') is retained from the dataset.

ples in Table 2. For each example, we also labeled surface patterns between the term and definition (e.g., "<term> defines <def>"), and potential algorithm improvements to address the underlying problem.

For instance, in the bottom-most example in Table 2 , the system did not predict any term or definition, although the sentence includes the term "Inductive Logic Programming Learning method" and the definition "extract from a corpus...". Our conjecture is that the underlying surface pattern is unseen in the training set and too complicated to be generalized; we annotate a potential solution as pattern generalization.

\begin{tabular}{rr}
\hline Top hypothesized causes of error & $\mathbf{( \% )}$ \\
\hline Overgeneralization: technical term bias & $48.6 \%$ \\
Unfamiliar or unseen vocabulary & $25.7 \%$ \\
Complicated sentence structure & $12.9 \%$ \\
Entity detection & $4.3 \%$ \\
\hline Overgeneralization: technical term bias & $28.9 \%$ \\
Overgeneralization: surface pattern bias & $23.3 \%$ \\
Unseen patterns & $14.4 \%$ \\
Complicated sentence structure & $12.2 \%$ \\
Overgeneralization: description & $3.3 \%$ \\
\hline
\end{tabular}

Table 3: Top causes of errors for terms (top) and definitions (bottom)

We rank the causes of errors by frequency and summarize the results in Table 3 . For detection of terms, nearly half of the error cases fall into overgeneralization of technical terms: overly predicting words like "equal" and "model" as terms (e.g., the top example in Table 2).

\begin{tabular}{rr}
\hline Proposed error correction solution types & $\mathbf{( \% )}$ \\
\hline Syntactic (POS, parse tree, entity, acronym) & $29.2 \%$ \\
Heuristics & $23.6 \%$ \\
Better encoder/tokenizer, UNK & $18.0 \%$ \\
Rules (surface patterns) & $11.3 \%$ \\
Annotations* & $9.4 \%$ \\
Pattern generalization & $5.7 \%$ \\
Mathematical symbol detection & $1.9 \%$ \\
More context & $0.9 \%$ \\
\hline
\end{tabular}

Table 4: Proportions of proposed error correction solution types. Annotations* indicates extremely ambiguous cases even for humans, so additional human annotations are required to disambiguate them.

We again rank the error correction solutions by frequency (Table 4). We predict that $29 \%$ of errors can be fixed by informing the system about syntactic features of the sentence such as part-of-speech tags, parse tree annotations, entities, or acronyms for more accurate detection. Surprisingly, simple heuristics (e.g., stitching up discontiguous token spans) seem likely to be highly effective to address the errors in Table 3, such as discarding output that does not successfully predict both a term and a definition. In the next section, we implement the first three solution types on top of the state-ofthe-art system and report the resulting performance improvements. 


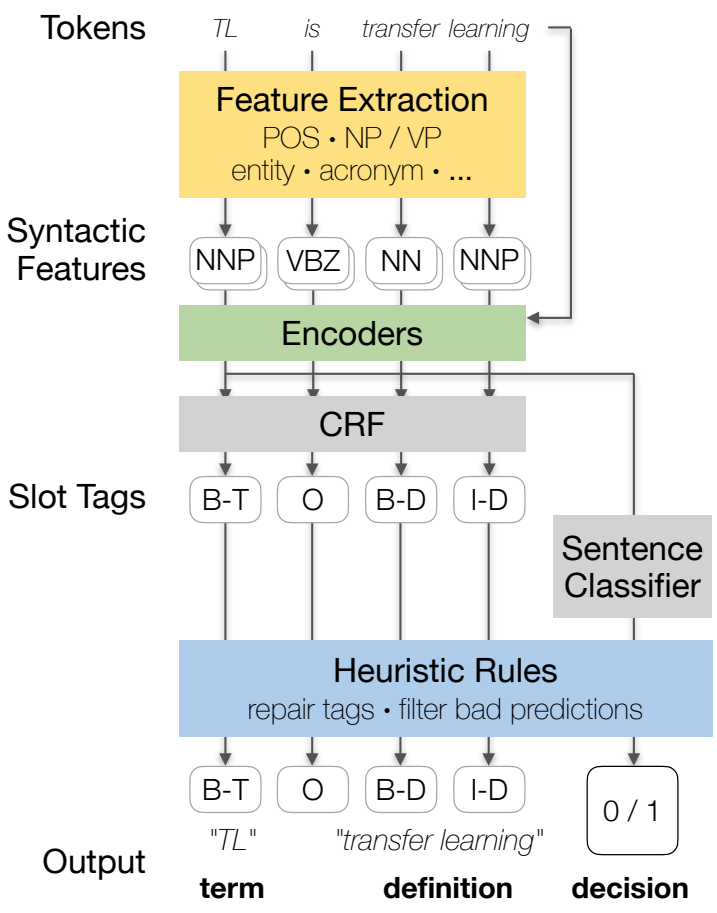

Figure 1: The HEDDEx model. The new modules developed in this work include the incorporation of syntactic features, the addition of pre-trained transformer encoders, and post-processing with heuristics.

\section{Definition Sentence Detection Model}

To address the errors identified in $\S 3$, we designed HEDDEx, a new sentence-level definition detection model. The model incorporates a set of syntactic features, heuristic filters, and encoders. Each of these was designed to address a common class of error revealed in the error analysis. The model achieves superior performance over the state of the art for the task of sentence-level definition detection.

\subsection{Proposed Model: Heuristically-Enhanced Deep Definition Extraction (HEDDEx)}

HEDDEx extends the joint model proposed by Veyseh et al. (2020). The joint model is comprised of two components. The first component is a CRFbased sequence prediction model for slot tagging. The model assigns each token in a sentence one of five tags: term ("B-TERM", "I-TERM"), definition ("B-DEF", "I-DEF"), or other ("O"). The second component is a binary classifier that labels each sentence as containing a definition or not.

HEDDEx has three new modules (Figure 1). First, it encodes input from a transformer encoder fine-tuned on the task of definition extraction, whereas the joint model encodes input from a combination of a graph convolutional network and a BERT encoder without fine-tuning. ${ }^{2}$ We evaluate several state-of-the-art encoders for this task, including BERT (Devlin et al., 2019), RoBERTa (Liu et al., 2019b), and SciBERT (Beltagy et al., 2019).

Second, HEDDEx is provided with additional syntactic features as input. These features include parts of speech, syntactic dependencies, and the token-level labels provided by entity recognizers and abbreviation detectors (Schwartz and Hearst, 2003). The features are extracted using off-theshelf tools like Spacy ${ }^{3}$ and SciSpacy (Neumann et al., 2019).

Third, the output of the CRF and sentence classifier is refined using heuristic rules. The rules clean up the slot tags produced by the CRF, and override predictions made by the sentence classifier. The rules include, among other rules:

- Do not classify a sentence as a definition if it only contains a term without a definition, or a definition without a term.

- Stitch up discontiguous token spans for terms and definitions by assigning all contiguous tokens between two term or definition labels the same label.

These three enhancements developed in HEDDEx were selected specifically to suit the shortcomings of the models identified in the error analysis (\$3), leading to significant improvements for definition detection in our experiments.

\subsection{Baseline Models}

To evaluate the impact of these improvements on the definition detection task, HEDDEx was compared to four baseline systems: (1) DefMiner (Jin et al., 2013), a CRF-based sequence prediction model that makes use of hand-written features; (2) Li et al.'s (2016) model comprised of a CRF with an LSTM encoder; (3) GCDT (Liu et al., 2019a), a global and local context encoder; (4) Veyseh et al.'s (2020) joint model described above. The experimental setup for the models followed the setup described by Veyseh et al. (2020).

\footnotetext{
${ }^{2}$ However, we note that we were unable to replicate the accuracy reported by Veyseh et al. (2020) when using the code provided by the authors.

${ }^{3}$ https://spacy.io/
} 


\subsection{Metrics}

The models were compared using a set of metrics for both slot tagging and sentence classification on the WOO test set. To evaluate the slot tagger, macro-averaged precision, recall, and F1 score were measured (column "Macro P/R/F" in Table 5). However, the Macro scores do not show performance specific to terms or definitions. Also, macroaveraging over the position tags (B, I) makes it difficult to interpret general performance. Therefore, we measured these three metrics only for term tags ("TERM P/R/F"); B-TERM and I-TERM, and definition tags ("DEF P/R/F"); B-DEF and I-DEF. To evaluate the precision of the bounds of term and definition spans, we also evaluated the degree of overlap between each detected term or definition span and the corresponding span in the gold dataset ("Partial F"). Furthermore, the accuracy of sentence classification was measured (column "Classification"). For each of these metrics, a higher score indicated superior performance. We averaged each score across 10 -fold cross validation.

\subsection{Setup}

Due to computing limitations, we chose the best hyper-parameter set through parameter sweeping with HEDDEx with the BERT encoder only, and use the best hyperparameters for all other models. Here is the ranges of each parameter we tuned: batch sizes in $\{8,16,32\}$, number of training epochs $[30,50,100]$ maximum length of sentences in $\{80,256,512\}$, learning rates in $[\{2,5\} \mathrm{e}-\{4,5\}]$.

The other parameters used as defaults in our experiments were as follows: the dropout ratio was $10 \%$, the layer size for POS embeddings was 50, and the hidden size for slot prediction was 512 . We follow the default hyper-parameters for each transformer model of each size (base or large) using HuggingFace's transformer libraries. ${ }^{4}$

\subsection{Results}

Outcomes of the evaluation for all measurements are presented in Table 5. The pre-trained language model encoders (BERT, RoBERTa, SciBERT) achieve comparable performance to more complex neural architectures like the graph convolutional networks used in Veyseh et al.'s (2020). Models that included SciBERT (Beltagy et al., 2019), rather than BERT or ROBERTa, achieved

\footnotetext{
${ }^{4}$ https://github.com/huggingface/ transformers
}

higher accuracy on most measurements. We attribute this to the domain similarity between the scholarly documents that SciBERT was trained on, and those used in our evaluation.

With SciBERT as the base encoder, the incorporation of syntactic features led to further accuracy gains. Of particular note are the improvements in recall in term spans. During our evaluation, we observed that the gains from syntactic features were more pronounced for encoders with a small mode size (i.e., the "-base" models). We conjecture that this is because the larger encoder models were capable of learning comparable linguistic patterns to those captured by the syntactic features.

The addition of heuristic rules led to significant improvement (+11.8 Macro F1) over the combination of Joint and SciBERT. Given the modest improvement in term and definition tagging, we suspect that much of this improvement can be accounted for by the correction of position markers in the slot tags (i.e., distinguishing between B and $\mathrm{I}$ in the tag assignments).

In the following experiments, we call HEDDEx the combination of three components: the encoder (SciBERT or RoBERTa), syntactic features, and heuristic filters.

\section{Document-Level Definition Detection}

Although HEDDEx attains reasonable performance on individual sentences, it faces new challenges when applied to the scenario of document-level analysis. In this section, we evaluate sentence detection for full papers in two novel ways. First, we assess the precision of the HEDDEx model across all of the sentences of 50 documents in $\$ 5.1$. Second, we assess both the precision and the recall of the algorithm across all of the sentences of 2 full documents (§5.2, S5.3).

\subsection{Error Analysis on Predicted Definitions}

To assess how well HEDDEx works at the document level, we randomly sampled 50 ACL papers from the S2ORC dataset (Lo et al., 2020), a large corpus of $81.1 \mathrm{M}$ English-language academic papers spanning many academic disciplines. We ran the pretrained HEDDEx model on every sentence of every document; if the model detected a term/definition pair, the corresponding sentence was output for assessment. (Note that this analysis can estimate precision but not recall, as false negatives are not detected.) 


\begin{tabular}{|c|c|c|c|c|c|}
\hline & Macro P/R/F & TERM P/R/F & DEF P/R/F & Partial F & Clsf. \\
\hline DefMiner (Jin et al., 2013) & $52.5 / 49.5 / 50.5$ & - & - & - & - \\
\hline LSTM-CRF (Li et al., 2016) & $57.1 / 55.9 / 56.2$ & - & - & - & - \\
\hline GCDT (Liu et al., 2019a) & $57.9 / 56.6 / 57.4$ & - & - & - & - \\
\hline Joint (Veyseh et al., 2020) & $60.9 / 60.3 / 60.6$ & - & - & - & - \\
\hline Joint* (Veyseh et al., 2020) & $61.0 / 60.2 / 60.7$ & - & - & - & 70.5 \\
\hline \multicolumn{6}{|l|}{ HEDDEx } \\
\hline Joint* + BERT-base & $59.5 / 61.3 / 60.3$ & $66.6 / 70.0 / 68.2$ & $72.1 / 74.0 / 72.8$ & 74.3 & 83.4 \\
\hline Joint* + BERT-large & 60.4 / 61.4 / 60.7 & $67.5 / 71.0 / 69.0$ & $72.3 / 73.9 / 72.9$ & 74.5 & 83.2 \\
\hline Joint* + RoBERTa-large & 60.3 / 61.6 / 60.7 & 67.3 / 70.3 / 68.6 & 72.8 / $74.6 / 73.5$ & 73.2 & 84.2 \\
\hline Joint $*$ + SciBERT & $61.9 / 61.2 / 61.5$ & $\mathbf{7 1 . 1} / 69.1$ / 69.9 & $74.0 / 74.6 / 74.2$ & 75.7 & 85.1 \\
\hline Joint* + SciBERT + Syntactic & $61.6 / 61.8 / 61.6$ & $70.7 / 71.3 / 70.9$ & $73.3 / 72.4 / 72.8$ & 74.3 & 84.3 \\
\hline Joint* + SciBERT + Syntactic + Heuristic & $72.9 / 74.3 / 73.4$ & $69.8 / 72.1 / 70.8$ & $75.4 / 71.8 / 73.3$ & 74.3 & 84.5 \\
\hline
\end{tabular}

Table 5: Comparison of the accuracy of recent models and the HEDDEx model for the definition detection task. Asterisks (*) indicate that we reimplemented the model from the authors' specification. Models were evaluated on the WOQ (Jin et al., 2013) test set. Each score is averaged across 10-fold cross validation. Accuracy measurements include Precision, Recall, and F1-score. Each of these measurements is macro-averaged.

We replace all citations and references to figures, tables, and sections with corresponding placeholders (e.g., CITATION, FIGURE), but keep raw TEX format of mathematical symbols in order to retain the structure of the equations. From the $50 \mathrm{ACL}$ papers, the model detected 924 definitions out of 13,658 sentences and the average number of definitions per paper is 18.5 .

\begin{tabular}{rrrr}
\hline Term (\%) & \multicolumn{2}{c}{ Definition (\%) } \\
\hline Textual term & $45.2 \%$ & Textual Def. & $58.7 \%$ \\
Incorrect term & $27.3 \%$ & Other: implausible & $24.8 \%$ \\
Math symbol term & $22.7 \%$ & Other: plausible & $11.8 \%$ \\
Acronym & $3.3 \%$ & Short name / Synonym & $3.5 \%$ \\
Acronym and text & $1.3 \%$ & Textual \& Formula Def. & $0.6 \%$ \\
& & Formula Def. & $0.4 \%$ \\
\hline
\end{tabular}

Table 6: Analysis of HEDDEx output on 50 ACL papers, ordered by frequency. $\mathrm{N}=923$.

The third author evaluated the predicted terms and definitions separately by choosing one among the labels shown in Table 6. For terms, the algorithm correctly labeled $72.5 \%$. We subdivide these correctly labeled terms into standard terms $(45.2 \%)$, math symbols $(22.7 \%)$, acronyms, acronym $(3.3 \%)$, or acronym and text $(1.3 \%)$. Among the correctly labeled definitions (total $63.2 \%=58.7 \%+3.5 \%+0.6 \%+0.4 \%), 92.6 \%$ are textual definitions, $5.6 \%$ are short names or synonyms, and $1.7 \%$ include mathematical symbols. We divided non-definitional text into two types: plausible (24.8\%) and implausible (11.8\%), which signals an error. The plausible text refers to explanations or secondary information (similar to DEFT (Spala et al., 2019)'s secondary definition, but without sentence crossings).

\begin{tabular}{rrrr}
\hline Term Span (\%) & \multicolumn{2}{r}{ Definition Span (\%) } \\
\hline Correct & $83.4 \%$ & Correct & $89.9 \%$ \\
Too Long (to the right) & $10.1 \%$ & Cut Off (to the right) & $3.4 \%$ \\
Cut Off (to the right) & $3.2 \%$ & Too Long (to the left) & $2.6 \%$ \\
Cut Off (to the left) & $1.6 \%$ & Cut Off (to the left) & $2.4 \%$ \\
Too Long (to the left) & $1.4 \%$ & Too Long (to the right) & $1.3 \%$ \\
\hline
\end{tabular}

Table 7: Analysis of span length of HEDDEx output on 50 ACL papers, ordered by frequency. $N=923$.

We also measured whether the predicted span length is correct, too long, or cut off (Table 7). These scores are quite high; $83.4 \%$ correct for terms and $89.9 \%$ for definitions (see Table 10).

\subsection{Full Document Definition Annotation}

Prior definition annotation collections select unrelated sentences from across a document collection. As mentioned in the introduction, we are interested in annotating full papers, which requires finding $\mathrm{ev}$ ery definition within a given paper. Therefore, we created a new collection in which we annotate every sentence within a document, allowing assessment of recall as well as precision. Two annotators annotated two full papers using an annotation scheme similar to that used in DEFT (Spala et al., 2019) except for omitting cross-sentence links.

We chose to annotate two award-winning ACL papers: ELMo (Peters et al., 2018) and LISA (Strubell et al., 2018) resulting in 485 total sentences from which we identified 98 definitional and 387 non-definitional sentences. Similar to DEFT (Spala et al., 2019), we measured inter-annotator agreement using Krippendorff's alpha (Krippendorff, 2011) with the MASI distance metric (Passonneau, 2006). We obtained 0.626 for terms and 
0.527 for definitions, where the agreement score for terms is lower than those in DEFT annotations $(0.80)$. This may be because our annotations for terms include various types such as textual terms, acronyms, and math symbols, while terms in DEFT are only textual terms. The task was quite difficult: each annotator takes two and half hours to annotate a single paper. Future work will include refining the annotation scheme to ensure more consistency among annotators and to annotate more documents.

\subsection{Evaluation on Document-level Definitions}

We evaluated document-level performance using the same metrics used in $\$ 4.3$. All metrics were averaged over scores from 10 -fold validation models. The ensemble model aggregates ten system predictions from the 10-fold validation models and choose the final label via majority voting. We use the best single system; HEDDEx but with RoBERTa, ${ }^{5}$ for model ensembling.

\begin{tabular}{|c|c|c|c|c|c|}
\hline & Macro & TERM & I DEF & Partial & Clf. \\
\hline & 50 & 301 & 38.1 & 34.1 & 86.8 \\
\hline & 4 & 32 & 39. & 3 & 89 \\
\hline $\mathrm{HE}$ & 47.7 & 36.4 & 47.2 & 37 & 88 \\
\hline HEDDEx ensemble & 50.4 & 38.7 & 49.5 & 39.0 & 89.8 \\
\hline
\end{tabular}

Table 8: Document-level evaluation on our annotated documents. F1 score is measured for every metric except for classification (Clf.), which uses accuracy.

Compared to the joint model by Veyseh et al. (2020), HEDDEx showed significant improvements on every evaluation metric, which is slightly larger than that of the sentence-level evaluation $(\mathrm{Ta}-$ ble 8). With model ensembling, compared to the state-of-the-art system, HEDDEx achieved gains by +14.4 Macro F1 points, +8.7 TERM F1 points, +11.4 DEF F1 points, +4.9 Partial Matching F1 points, and +3.0 classification accuracy scores.

\begin{tabular}{lccc}
\hline & Precision & Recall & F1 \\
\hline Macro & 55.3 & 46.7 & 50.4 \\
TERM & 44.8 & 34.0 & 38.7 \\
DEF & 55.6 & 44.7 & 49.5 \\
\hline
\end{tabular}

Table 9: Low recall problem in document-level definition detection. We report precision, recall, and f1 scores on three metrics; Macro, TERM, and DEF, using our best system; HEDDEx ensemble.

However, document-level definition detection is

\footnotetext{
${ }^{5}$ RoBERTa and SciBERT show comparable performance on the document-level definition detection task.
}

a much harder task than sentence-level detection. Compared to the sentence-level task in Table 5, the document-level task showed relatively lower performance (73.4 Macro F1 in sentence-level versus 50.4 Macro F1 in document-level). In particular, recall is much lower than precision in the documentlevel task (Table 9), whereas in the sentence-level task, precision and recall are almost the same, indicating the necessity of incorporation of document structure as additional features (See further discussion in §6).

Table 10 shows the predicted terms and definitions as well as annotated gold labels. Acronym patterns (e.g., "biLM," "WSD"), definition of newlyproposed terms (e.g., "LISA"), re-definition of prior work (e.g., "SQuAD," "SRL," Coreference resolution) and some of mathematical symbols were detected well. However, as sentences get more complex, the system made incorrect predictions. Additionally, sub-words or parentheses in abbreviations are sometimes partially predicted (e.g., the beginning of the word "pretrained" is cut off in the definition of "semi-supervised learning" in example 8 of Table 10) .

However, the aforementioned problem of low recall is severe for this task, particularly since the model often fails to detect mathematical symbols or a combination of textual terms and mathematical symbols (e.g., " $L$-layer biLM"). Moreover, when a sentence contains multiple terms and/or multiple symbols together, the system only ever detects one of them.

\section{Discussion}

Detecting definitions is a very challenging task, and it is far from solved. Here we discuss remaining challenges and ideas for improvements, and motivate the need for high-precision, high-recall definition detection in an academic document reading aid application.

Outstanding technical challenges include:

\section{- Poor recognition of mathematical symbols:} As shown in our experiment, our system is less successful at detecting math symbols than textual terms. This is mainly because the lack of coverage of mathematical symbols in our training dataset $(W 00)$.

- Contextual disambiguation of symbols: In our study, we observe that some symbols are used with multiple meanings. For example, 


\begin{tabular}{|c|c|c|}
\hline & Predicted definition sentences & Type \\
\hline & $\begin{array}{l}\text { Our word vectors are learned functions of the internal states of a \{deep bidirectional language model] ([biLM]), } \\
\text { which is pre-trained on a large text corpus. }\end{array}$ & term \\
\hline & e use vectors derived from & \\
\hline
\end{tabular}

3 Using intrinsic evaluations, we show that the higher-level [LSTM states] capture context-dependent aspects of term word meaning (e.g., they can be used without modification to perform well on supervised word sense disambiguation tasks) while lower-level states \{model aspects of syntax\}.

4 We first show that they can be easily added to existing models for six diverse and challenging language term understanding problems, including textual entailment, question answering and sentiment analysis.

5 For tasks where direct comparisons are possible, outperforms [CoVe] CITATION, which term \{computes contextualized representations using a neural machine translation encoder].

6 context2vec CITATION uses a bidirectional Long Short Term Memory LSTM ; CITATION to encode the context term around a pivot word.

7 Unlike most widely used word embeddings CITATION, word representations are functions of the entire input term sentence, as described in this section.

8 This setup allows us to do [semi-supervised learning], where the biLM is pretr\{ained at a large scale\} (Sec. term SECTION) and easily incorporated into a wide range of existing neural NLP architectures (Sec. SECTION).

9 Given a sequence of $N$ tokens, $\left(t_{1}, t_{2}, \ldots, t_{N}\right)$, a forward language model computes the probability of the sequence term by modeling the probability of token $t_{k}$ given the history $\left(t_{1}, \ldots, t_{k-1}\right)$ :

10 A [backward LM] is \{ similar to a forward LM, except it runs over the sequence in reverse, predicting the term previous token given the future context $]$ :

11 A [biLM] \{combines both a forward and backward $L M\} . \quad$ term

12 where $\left[h_{k, 0}^{L M}\right]$ is $\{$ the token layer $\}$ and $h_{k, j}^{L M}=\left[\vec{h}_{k, j}^{L M} ; \overleftarrow{h}_{k, j}^{L M}\right]$, for each biLSTM layer. symbol

13 In (EQUATION), $\left[s^{\text {task }}\right]$ are $\{$ softmax-normalized weights $\}$ and the scalar parameter $\gamma^{\text {task }}$ allows the task model to symbol scale the entire vector.

14 For each token $t_{k}$, a $L$-layer biLM computes a set of $2 L+1$ representations EQUATION where $h_{k, 0}^{L M}$ is the token symbol, layer and $h_{k, j}^{L M}=\left[\vec{h}_{k, j}^{L M} ; \overleftarrow{h}_{k, j}^{L M}\right]$, for each biLSTM layer.

15 In (EQUATION), $\underline{s}^{\text {task }}$ are softmax-normalized weights and the [scalar parameter $\gamma^{\text {task }}$ ] \{allows the task model symbol to scale the entire vector $\}$.

16 The [Stanford Question Answering Dataset (SQuAD) CITATION] \{contains 100K+_crowd sourced term question-answer pairs where the answer is a span in a given Wikipedia paragrap $\bar{h} \bar{h} \bar{\gamma}$.

17 [Textual entailment] is \{the task of determining whether a "hypothesis" is true, given a "premise" $\}. \quad$ term

18 The [Stanford Natural Language Inference (SNLI) corpus CITATION] \{provides approximately 550K hy- term pothesis/premise pairs\}.

19 A [semantic role labeling (SRL) system] \{models the predicate-argument structure of a sentence\}, and is often term described as answering.

20 CITATION modeled [SRL] \{as a BIO tagging problem and used an 8-layer deep biLSTM with forward and term backward directions interleaved $\}$, following CITATION.

21 [Coreference resolution] is $\{$ the task of clustering mentions in text that refer to the same underlying real world term entities\}.

22 The [CoNLL] 2003 NER task CITATION \{consists of newswire from the Reuters RCV1 corpus tagged with four term different entity types (PER, LOC, ORG, MISC)\}.

23 The [fine-grained sentiment classification] task in the Stanford Sentiment Treebank SST-5 \{involves selecting term one of five labels (from very negative to very positive) to describe a sentence from a movie review\}.

24 The sentences contain diverse linguistic phenomena such as idioms and complex syntactic constructions such as multinegations that are difficult for models to learn.

25 Intuitively, the [biLM] must be \{disambiguating the meaning of words using their context\}.

26 a fine grained \{word sense disambiguation\} ([WSD]) task and a POS tagging task. term

Table 10: All predicted and gold label terms and definitions for the ELMo paper (Peters et al., 2018). Gold labels for terms are underlined and for definitions are dashed. System-predicted [terms] are placed in [boldfaced

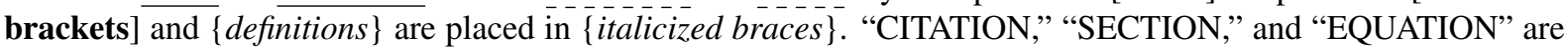
placeholders inserted for citations, section numbers, and 2 dissplay equations. "Type" means term type. 
symbol $T$ in the LISA paper is used for token representation as well as matrix transpose. Disambiguating terms based on context of use will be an interesting future direction.

- Description vs Definition: In our annotation and error analysis, the most difficult distinction was between definitions and descriptions - they have quite similar surface patterns, although they refer to entirely different meanings. For instance, a definition is the exact denotation of a word, while a description is more detailed so it can change from person to person. Training a model that distinguishes these types should lead to better and more useful results.

Potential ideas for improvements of the system include:

- Annotation of mathematical definitions: A solution for poor math symbol detection is to annotate math symbols and use them for our training. One option is to add span information to the binary judgements of the math definition collection of Vanetik et al. (2020).

- Utilization of document-level features: Document structure and positional information may improve detection. For instance, the section information of a term would be an important feature to recognize whether a term is first introduced or not.

- Data augmentation or domain-specific fine-tuning for high-recall system: Existing definition training sets are small (WOQ contains only 731 definitional sentences). To obtain more data, the data can be augmented via seed patterns or fine-tuning with existing language models such as SciBERT.

Lastly, as the performance of definition detection systems increases, these systems can be applied to real-world reading or writing aids. We discuss potential issues of our system in the realistic settings:

- Metrics for usefulness: Currently, we measure precision, recall, and F1 scores with the document-level annotations. However, we have not explored the usefulness of the predicted definitions for readability, when they are used in real-world applications like ScholarPhi (Head et al., 2020). Deciding when and where to show definitions based on context and information density still remains an important future direction.
- Categorization of definitions: We observe that in fact, terms and definitions can be grouped into multiple categories: short names, acronyms, textual definitions, formula definitions, and more. Automatically categorizing these and showing structured definitions might be helpful for organizing and ranking definitions in a user interface.

- Repeated definitions and terms within documents: We observed a pattern in which the same term is referred to multiple times in slightly different ways. Newly proposed terms are especially likely to exhibit this pattern. Grouping and summarizing these in a glossary table would be helpful for an academic document reader application.

\section{Conclusion}

This work sets the stage for bridging the gap between a well-known NLP task; definition detection, and real-world applications of the technique that requires both high precision and high recall. To achieve the goal, we proposed a more realistic setup for definition detection task called documentlevel definition detection that requires high recall, mathematical symbol recognition, and documentlevel feature engineering. Our proposed definition detection system HEDDEx achieved significant gains in both sentence-level and document-level tasks. Yet, the problem is far from being solved. We suggest that better coverage of variability of expression, recognition of mathematical symbols and notation, and other nuances of the task must still be addressed.

\section{Acknowledgements}

We like to thank Amir Pouran Ben Veyseh for his help in sharing his code, preprocessed data, and general advice on replication of his work. We also thank Raymond Fok, Vivek Aithal, Hearst lab members at UC Berkeley and anonymous reviewers at SDP 2020 for their helpful comments. This research receives funding from the Alfred P. Sloan Foundation, the Allen Institute for AI, Office of Naval Research grant N00014-15-1-2774, NSF Convergence Accelerator award 1936940, NSF RAPID award 2040196, and the University of Washington Washington Research Foundation/Thomas J. Cable Professorship. 


\section{References}

Satanjeev Banerjee and Ted Pedersen. 2002. An adapted lesk algorithm for word sense disambiguation using wordnet. In International conference on intelligent text processing and computational linguistics, pages 136-145. Springer.

Iz Beltagy, Kyle Lo, and Arman Cohan. 2019. Scibert: A pretrained language model for scientific text. In Proceedings of the 2019 Conference on Empirical Methods in Natural Language Processing and the 9th International Joint Conference on Natural Language Processing (EMNLP-IJCNLP), pages 36063611 .

Claudio Delli Bovi, Luca Telesca, and Roberto Navigli. 2015. Large-scale information extraction from textual definitions through deep syntactic and semantic analysis. Transactions of the Association for Computational Linguistics, 3:529-543.

Jacob Devlin, Ming-Wei Chang, Kenton Lee, and Kristina Toutanova. 2019. BERT: Pre-training of deep bidirectional transformers for language understanding. In Proceedings of the 2019 Conference of the North American Chapter of the Association for Computational Linguistics: Human Language Technologies, Volume 1 (Long and Short Papers), pages 4171-4186, Minneapolis, Minnesota. Association for Computational Linguistics.

Luis Espinosa-Anke and Steven Schockaert. 2018. Syntactically aware neural architectures for definition extraction. In Proceedings of the 2018 Conference of the North American Chapter of the Association for Computational Linguistics: Human Language Technologies, Volume 2 (Short Papers), pages 378-385, New Orleans, Louisiana. Association for Computational Linguistics.

Ismail Fahmi and Gosse Bouma. 2006. Learning to identify definitions using syntactic features. In Proceedings of the Workshop on Learning Structured Information in Natural Language Applications.

Chikara Hashimoto, Kentaro Torisawa, Stijn De Saeger, Sadao Kurohashi, et al. 2011. Extracting paraphrases from definition sentences on the web. In Proceedings of the 49th Annual Meeting of the Association for Computational Linguistics: Human Language Technologies, pages 1087-1097.

Andrew Head, Kyle Lo, Dongyeop Kang, Raymond Fok, Sam Skjonsberg, Daniel S. Weld, and Marti A Hearst. 2020. Augmenting scientific papers with just-in-time, position-sensitive definitions of terms and symbols. arXiv preprint arXiv:2009.14237.

Luyao Huang, Chi Sun, Xipeng Qiu, and Xuanjing Huang. 2019. GlossBERT: BERT for word sense disambiguation with gloss knowledge. In Proceedings of the 2019 Conference on Empirical Methods in Natural Language Processing and the 9th International Joint Conference on Natural Language Processing (EMNLP-IJCNLP), pages 3509-3514, Hong
Kong, China. Association for Computational Linguistics.

Yiping Jin, Min-Yen Kan, Jun-Ping Ng, and Xiangnan He. 2013. Mining scientific terms and their definitions: A study of the ACL anthology. In Proceedings of the 2013 Conference on Empirical Methods in Natural Language Processing, pages 780-790, Seattle, Washington, USA. Association for Computational Linguistics.

Klaus Krippendorff. 2011. Computing krippendorff's alpha-reliability. Technical report, University of Pennsylvania. Retrieved from https://repository.upenn.edu/asc_papers/43/.

John D. Lafferty, Andrew McCallum, and Fernando C. N. Pereira. 2001. Conditional random fields: Probabilistic models for segmenting and labeling sequence data. In Proceedings of the Eighteenth International Conference on Machine Learning, pages 282-289, San Francisco, CA, USA. Morgan Kaufmann Publishers Inc.

SiLiang Li, Bin Xu, and Tong Lee Chung. 2016. Definition extraction with lstm recurrent neural networks. In Chinese Computational Linguistics and Natural Language Processing Based on Naturally Annotated Big Data, pages 177-189. Springer.

Yijin Liu, Fandong Meng, Jinchao Zhang, Jinan Xu, Yufeng Chen, and Jie Zhou. 2019a. GCDT: A global context enhanced deep transition architecture for sequence labeling. In Proceedings of the 57th Annual Meeting of the Association for Computational Linguistics, pages 2431-2441, Florence, Italy. Association for Computational Linguistics.

Yinhan Liu, Myle Ott, Naman Goyal, Jingfei Du, Mandar Joshi, Danqi Chen, Omer Levy, Mike Lewis, Luke Zettlemoyer, and Veselin Stoyanov. 2019b. Roberta: A robustly optimized bert pretraining approach. arXiv preprint arXiv:1907.11692.

Kyle Lo, Lucy Lu Wang, Mark Neumann, Rodney Kinney, and Daniel Weld. 2020. S2ORC: The semantic scholar open research corpus. In Proceedings of the 58th Annual Meeting of the Association for Computational Linguistics, pages 4969-4983, Online. Association for Computational Linguistics.

A Muresan and Judith Klavans. 2002. A method for automatically building and evaluating dictionary resources. In Proceedings of the Language Resources and Evaluation Conference (LREC.

Roberto Navigli and Paola Velardi. 2010. Learning word-class lattices for definition and hypernym extraction. In Proceedings of the 48th Annual Meeting of the Association for Computational Linguistics, pages 1318-1327, Uppsala, Sweden. Association for Computational Linguistics.

Mark Neumann, Daniel King, Iz Beltagy, and Waleed Ammar. 2019. Scispacy: Fast and robust models for 
biomedical natural language processing. In Proceed ings of the 18th BioNLP Workshop and Shared Task, pages 319-327.

Rebecca J Passonneau. 2006. Measuring agreement on set-valued items (masi) for semantic and pragmatic annotation. In Proceedings of the Fifth International Conference on Language Resources and Evaluation (LREC'06).

Matthew Peters, Mark Neumann, Mohit Iyyer, Matt Gardner, Christopher Clark, Kenton Lee, and Luke Zettlemoyer. 2018. Deep contextualized word representations. In Proceedings of the 2018 Conference of the North American Chapter of the Association for Computational Linguistics: Human Language Technologies, Volume 1 (Long Papers), pages 22272237.

Melanie Reiplinger, Ulrich Schäfer, and Magdalena Wolska. 2012. Extracting glossary sentences from scholarly articles: A comparative evaluation of pattern bootstrapping and deep analysis. In Proceedings of the ACL-2012 Special Workshop on Rediscovering 50 Years of Discoveries, pages 55-65, Jeju Island, Korea. Association for Computational Linguistics.

Ariel S. Schwartz and Marti A. Hearst. 2003. A simple algorithm for identifying abbreviation definitions in biomedical text. Pacific Symposium on Biocomputing. Pacific Symposium on Biocomputing, pages 451-62.

Sasha Spala, Nicholas A. Miller, Yiming Yang, Franck Dernoncourt, and Carl Dockhorn. 2019. DEFT: A corpus for definition extraction in free- and semistructured text. In Proceedings of the 13th Linguistic Annotation Workshop, pages 124-131, Florence, Italy. Association for Computational Linguistics.

Emma Strubell, Patrick Verga, Daniel Andor, David Weiss, and Andrew McCallum. 2018 Linguistically-informed self-attention for semantic role labeling. In Proceedings of the 2018 Conference on Empirical Methods in Natural Language Processing, pages 5027-5038, Brussels, Belgium. Association for Computational Linguistics.

Natalia Vanetik, Marina Litvak, Sergey Shevchuk, and Lior Reznik. 2020. Automated discovery of mathematical definitions in text. In Proceedings of The 12th Language Resources and Evaluation Conference, pages 2086-2094, Marseille, France. European Language Resources Association.

Amir Pouran Ben Veyseh, Franck Dernoncourt, Dejing Dou, and Thien Huu Nguyen. 2020. A joint model for definition extraction with syntactic connection and semantic consistency. In Thirty-Fourth AAAI Conference on Artificial Intelligence (AAAI-20).

Eline Westerhout. 2009a. Definition extraction using linguistic and structural features. In Proceedings of the 1st Workshop on Definition Extraction, pages 6167.
Eline Westerhout. 2009b. Extraction of definitions using grammar-enhanced machine learning. In Proceedings of the Student Research Workshop at EACL 2009, pages 88-96, Athens, Greece. Association for Computational Linguistics.

Eline Westerhout and Paola Monachesi. 2008. Creating glossaries using pattern-based and machine learning techniques. In LREC 2008. 